\title{
Finding multiplies solutions for non-linear integer programming
}

\author{
José Manuel Jiménez Cobano ${ }^{2}$ \\ Instituto de Matemática \\ Universidad de Sevilla \\ José María Ucha Enríquez ${ }^{1,3}$ \\ Departamento de Matemática Aplicada I \\ Universidad de Sevilla
}

\begin{abstract}
We explain how to compute all the solutions of a nonlinear integer problem using the algebraic test-sets associated to some linear subproblem. These test-sets are obtained using Gröbner bases. We compare our method with previous approaches . Keywords: Integer non-lineal optimization, multiplies solutions, test-sets, Groebner basis

1 Second author is partially funded by MTM2016-75024-P, P12-FQM-2696, MTM201674983-C2-1-R and FQM-333.

2 Email: josjimcob@alum.us.es

3 Email: ucha@us.es
\end{abstract}




\section{Introduction}

In many real-life combinatorial optimization problems is of great interest for the decision-maker to have not only one solution, but the set of all optimal solutions (see [10] or [7], for example). The information provided by this set can give some additional information about the solutions, and sometimes is a first step for multi-objective optimization. On the other hand, sometimes these problems requires non-linear constraints to be modeled properly.

A method for problems of the form

$$
\begin{aligned}
\min & c x^{t} \\
\text { s.t. } & A x^{t} \leq b^{t} \\
& x \in \Omega \\
& x \in \mathbb{N}^{n}
\end{aligned}
$$

where $A \in \mathbb{Z}^{m \times n}, c \in \mathbb{Z}^{n}, b \in \mathbb{Z}^{m}$ and the region $\Omega$ is defined by linear and nonlinear constraints was proposed in [9]. This method makes use of the test-sets associated to the linear subproblem

$$
\begin{aligned}
& \min c x^{t} \\
& \text { s.t. } A x^{t} \leq b^{t} \quad\left(P_{L}\right) \\
& \quad x \in \mathbb{N}^{n}
\end{aligned}
$$

A set $T \subset \mathbb{Z}^{n}$ is a test-set associated to $\left(P_{L}\right)$ if $T \subset \operatorname{ker}(A)$, and for any non optimal $x$ feasible for $P_{L}$ there exists a $t \in T$ such that $x-t$ is feasible and $c(x-t)<c(x)$. As a consequence, starting from a optimal point $\hat{x}$ of $\left(P_{L}\right)$ you can recover the set of all the feasible points, adding elements of the testset until you eventually complete all the feasible region. In this way you can obtain the optimal points of $(P)$ walking back from the linear optimal point until you reach the region $\Omega$. Technical details can be found in [9]. There are several ways of computing test-sets, and one of the most efficient is using Gröbner bases (see for example [3]) with the software 4ti2 (see [5]).

In [1] and [6] the method of [9] is applied to real-life size problems with very competitive results. In this work: 1) we explain how to modify the walk-back method to obtain all the optimal points and 2) we compare its performance with the natural generalization of the algorithm presented in [10] using the computational system COUENNE (see [2]). 


\section{An algebraic algorithm to find all optimal points.}

The main idea of our method is quite simple: starting from optimal point of the linear problem $\left(P_{L}\right)$, we add test-set vectors until we find the points inside the non-linear region $\Omega$. We save all points that has the best cost into $\Omega$. The pseudocode is the following one:

INPUT: $c, A, b ; \Omega$; optimal point $\beta$ of $P_{L} ; T$ associated test-set of $P_{L}$.

Opt $:=\emptyset$

Leaves $:=\{\beta+t \mid \forall t \in T\} \cap \mathbb{N}^{n}$

$\operatorname{costOpt}=\infty$

IF $\beta \in \Omega$

THEN $O p t:=\{\beta\}$;

$\operatorname{costOpt}:=c \beta^{t}$

WHILE $($ Leaves $\neq \emptyset)$ DO

FOR $h \in$ Leaves DO

IF $c(h)<\operatorname{costOpt}$

Leaves $=($ Leaves $\backslash\{h\}) \cup\left(\{h+t \mid \forall t \in T\} \cap \mathbb{N}^{n}\right)$

IF $h \in \Omega$

THEN $O p t=\{h\}$

$\operatorname{costOpt}=\operatorname{ch}^{t}$

Leaves $=($ Leaves $\backslash\{h\}) \cup\left(\{h+t \mid \forall t \in T\} \cap \mathbb{N}^{n}\right)$

$\sharp$ the list of old candidates is deleted

$\sharp$ and updated with a new candidate

ELSE IF $c(h)>\operatorname{costOpt}$

THEN Leaves $=$ Leaves $\backslash\{h\}$

$\sharp$ these branches are discarded

ELSE IF $c(h)=\operatorname{costOpt}$

THEN Leaves $=($ Leave $\backslash\{h\}) \cup\left(\{h+t \mid \forall t \in T\} \cap \mathbb{N}^{n}\right)$

IF $h \in \Omega$

THEN $O p t=O p t \cup\{h\}$;

$\sharp$ a new candidate to be an optimal point has been obtained

END WHILE

OUTPUT: Opt the set of all optimal points with cost costeOpt 


\section{Computational experiments: reliability of series-parallel systems}

Reliability problems are considered an important measure in the design of engineering processes, in which a series of systems is similar to a chain in which all the components must operate, since the failure of one of these components will suppose the failure of the complete system.

A mathematical model to minimize the cost of the system is

(1)

$$
\begin{array}{ll}
\min & \sum_{i=0}^{n} \sum_{j=1}^{k} c_{i j} x_{i j} \\
\text { s.t. } & R(x) \geq R_{0} \\
& \sum_{j=1}^{k} x_{i j} \geq 1, \forall i=1, \ldots, n \\
& 0 \leq x_{i j} \leq u_{i j}, \forall i=1, \ldots, n, j=1, \ldots, k \\
& x_{i j} \in \mathbb{Z}^{+}
\end{array}
$$

with $R(x)=\prod_{i=1}^{n}\left(1-\prod_{j=1}^{k}\left(1-r_{i j}\right)^{x_{i j}}\right)$. Where:

- $n$ : number of subsystems

- $k_{i}$ : number of different kinds of components in subsystem $i$.

- $r_{i j}$ : reliability of component $j$ in the subsystem $i$.

- $c_{i j}$ : cost of component $j$ in the subsystem $i$.

- $l_{i j}, u_{i j}$ : upper and lower dimensions of the numbers of components $j$ in the subsystem $i$. We can suppose that $l_{i j}=0$.

- $R_{0}$ : Minimum reliability required return for the whole system.

- $x_{i j}$ : numbers of components $j$ in the subsystem $i$.

In our experiments, we have considered that all subsystem have equal numbers of components, that is, $k_{i}=k, \forall i$.

- $r_{i j} \in(0.9,0.99), \forall i=1, \ldots, n, j=1, \ldots, k$

- $c_{i j} \in\{1,2, \ldots, 10\}, \forall i=1, \ldots, n, j=1, \ldots, k$

- $R_{0}=0.9$

- $u_{i j}=4, \forall i=1, \ldots, n, j=1, \ldots, k$

- $n \in\{3,4\}$ 
- $k \in\{2,3\}$

We have run about 120 examples to test our algorithm coded in Python in a computer with an Intel Core i5, 3.5 Ghz, $8 \mathrm{~Gb}$ of RAM, under Ubuntu. The General Cut row stands for the natural generalization of the method proposed in [10], we have been used COUENNE ([2]) for the example which have been sent to neos-server.org.

\begin{tabular}{|c|c|c|}
\hline & Number of examples & $\%$ examples \\
\hline Test-set & 84 & $70 \%$ \\
\hline General cut & 36 & $30 \%$ \\
\hline
\end{tabular}

Table 1

Reliability examples $n=3,4, k=2,3$ with all solutions.

We can observe that:

\begin{tabular}{|c|c|c|}
\hline & Number of examples & $\%$ examples \\
\hline Test-set & 42 & $97,67 \%$ \\
\hline General cut & 1 & $2,33 \%$ \\
\hline
\end{tabular}

Table 2

Reliability examples $n=3,4, k=2,3$ with multiplies solutions.

\begin{tabular}{|c|c|c|}
\hline & Number of examples & $\%$ examples \\
\hline Test-set & 42 & $54,55 \%$ \\
\hline General cut & 35 & $45,45 \%$ \\
\hline
\end{tabular}

Table 3

Reliability examples $n=3,4, k=2,3$ an unique solution.

\begin{tabular}{|c|c|c|c|c|}
\hline & \multicolumn{2}{|c|}{3 Systems } & \multicolumn{2}{c|}{4 Systems } \\
\hline & 2 components & 3 components & 2 components & 3 components \\
\hline Test-set & 0,07 & 0,19 & 0,37 & 0,51 \\
\hline General cut & 0,43 & 1,23 & 0,84 & 1,50 \\
\hline
\end{tabular}

Table 4

Average CPU times Reliability examples $n=3,4, k=2,3$. 
- The Test-Set method and the General Cut are both exact

- The General Cut is worse in CPU time than the test-set method.

\section{References}

[1] Castro, F. J.; Gago, J.; Hartillo, I.; Puerto, J.; Ucha, J. M. An algebraic approach to integer portfolio problems. European J. Oper. Res. 210 (2011), no. $3,647-659$.

[2] Belotti, Pietro. COUENNE: a user's manual. Document available at https://www. coin-or.org/Couenne/. https://www.coin-or.org/Couenne/

[3] Cox, David A.; Little, John; O'Shea, Donal. Using algebraic geometry. Second edition. Graduate Texts in Mathematics, 185. Springer, New York, 2005.

[4] Djerdjour M, Rekab K. A branch and bound algorithm for designing reliable systems at a minimum cost. Appl. Math. and Computation 118 (2001), 247-59.

[5] 4ti2 team. 4ti2 - A software package for algebraic, geometric and combinatorial problems on linear spaces. Available at www.4ti2.de, 2013.

[6] Gago, J.; Hartillo, I.; Puerto, J.; Ucha, J. M. Exact cost minimization of a seriesparallel reliable system with multiple component choices using an algebraic method. Comput. Oper. Res. 40 (2013), no. 11, 2752-2759.

[7] Leão, Aline A. S.; Cherri, Luiz H.; Arenales, Marcos N. Determining the K-best solutions of knapsack problems. Comput. Oper. Res. 49 (2014), 71-82.

[8] Ruan N, Sun XL. An exact algorithm for cost minimization in series reliability systems with multiple component choices. Appl. Math. and Computation 181 (2006), 732-41.

[9] Tayur, Sridhar R.; Thomas, Rekha R.; Natraj, N. R. An algebraic geometry algorithm for scheduling in presence of setups and correlated demands. Math. Programming 69 (1995), no. 3, Ser. A, 369-401.

[10] Tsai, Jung-Fa; Lin, Ming-Hua; Hu, Yi-Chung Finding multiple solutions to general integer linear programs. European J. Oper. Res. 184 (2008), no. 2, 802809.

[11] Han-Lin Li, Tsai, Jung-Fa. A distributed computation algorithm for solving portfolio problems with integer variables. European J. Oper. Res. 186 (2008), no. $2,882-891$. 